\title{
Critical Limology - ein Ansatz kritischer Grenzforschung
}

\section{Thomas Nail}

\begin{abstract}
Im vorliegenden Beitrag wird die Critical Limology vorgestellt - eine neue, an Prozessen und Bewegungen orientierte Methodologie zur Erforschung von Grenzen. Dieser Ansatz erlaubt es, umfassende Muster wiederkehrender (zirkulärer) Bewegungen (patterns of circulation) über längere historische Perioden und größere geografische Räume hinweg zu untersuchen, als es derzeit üblich ist. Er ermöglicht auch, die Dynamik von Grenzen selbst zu verstehen, die unter dem Einfluss dieser wiederkehrenden Bewegungen Veränderungen unterworfen sind. Der Beitrag zielt insbesondere darauf ab, aufzuzeigen, inwiefern dieser methodologische Ansatz Probleme der Grenzforschung lösen kann.
\end{abstract}

\section{Schlagwörter}

Grenztheorie, Kritische Theorie, Materialismus, Politik der Bewegung, Politische Theorie

\section{Einleitung}

In diesem Beitrag soll eine neue an Prozessen und Bewegungen orientierte Methodologie zur Erforschung von Grenzen vorgestellt werden: Ich nenne sie Critical Limology. Der Vorteil dieses Ansatzes besteht darin, dass er uns erlaubt, umfassende Muster wiederkehrender (zirkulärer) Bewegungen (patterns of circulation) über längere historische Perioden und größere geografische Räume hinweg zu untersuchen, als es derzeit üblich ist. Er ermöglicht uns auch, die Dynamik von Grenzen selbst zu verstehen, die unter dem Einfluss dieser wiederkehrenden Bewegungen Veränderungen unterworfen sind. Mein Beitrag zielt insbesondere darauf ab, aufzuzeigen, inwiefern dieser methodologische Ansatz zwei bedeutende Probleme der Grenzforschung lösen kann.

Das erste Problem besteht darin, dass Grenzen heute zunehmend „allgegenwärtig“ und deshalb mit den herkömmlichen Methoden nur schwer zu erfassen sind (Balibar 1998, S. 216 229). Deshalb läuft die zeitgenössische Grenztheorie derzeit Gefahr, vollständig in der politischen Theorie aufzugehen. Dies ist ein weithin erkanntes Dilemma unter Forschenden (vgl. Johnson et al. 2011, S. 62). Grenzforschung heute bewegt sich also im Spannungsfeld zwischen der Notwendigkeit einer allgemeineren theoretischen Basis einerseits, mit der die wachsende historische und empirische Bandbreite an Grenzen verstanden werden kann, und dem Anliegen andererseits, eine Generalisierung zu vermeiden, die die Besonderheiten spezifischer Grenzen außer Acht lässt. Was ich als Critical Limology bezeichne, stellt einen dritten methodologischen Ansatz zwischen einer allgemeinen ,Theorie‘ der Grenze und der streng empirischen Untersuchung einzelner Grenzen dar.

Ich führe in diesem Beitrag eine Methodologie ein, die nicht darauf ausgerichtet ist, universelle oder partikuläre Eigenschaften in den Blick zu nehmen (vgl. Gerst/Krämer in diesem Band). Sie soll vielmehr ergründen, was ich als historische Grenzregime oder als kinetische Muster der Bewegung, auf die Grenzen zurückgeführt werden können, bezeichnen möchte. Unterschiedliche Grenztechnologien bringen im Laufe der Geschichte verschiedene Regime oder Muster wiederkehrender sozialer Bewegungen hervor. Wenn diese Grenztechnologien einmal erfunden 
worden sind, haben ihre Bewegungsmuster in der Regel bis in die Gegenwart hinein Bestand, solange wir fortfahren, ähnliche physische Technologien wie Zäune, Mauern, Zellen und Grenzübergänge als Methoden der Mobilitätskontrolle einzusetzen. Kritische Grenzforschung untersucht die Entstehung und Zusammensetzung dieser historischen Strukturen der Grenzerhaltung, ohne den Anspruch zu erheben, eine universelle Theorie der Grenze zu sein oder umgekehrt Grenzen auf ihre spezifischen Merkmale zu reduzieren.

Das zweite Problem, um das es in diesem Beitrag geht, gründet darauf, dass Grenzforschung noch immer an das Konzept des modernen Staates gebunden zu sein scheint. Auf diese Weise wird Grenze auf einen ganz spezifischen historischen Nutzen reduziert. Dies trifft in zweierlei Hinsicht zu: Erstens wird, sofern Grenze als Objekt und nicht als Prozess betrachtet wird, die Stasis des Staates fälschlicherweise als Modell genutzt, um das Bestehen von Grenzen herzuleiten. Tatsächlich aber ist das Gegenteil der Fall. Prozesse der Grenzziehung gehen aus historischer Perspektive vielmehr dem Staat voraus und haben sein Aufleben erst ermöglicht. Das bedeutet, dass weder Staaten noch Grenzen statische Phänomene sind, sondern stattdessen als Prozesse fortdauernder und konstanter Zirkulation betrachtet werden sollten. Zweitens scheint die Grenzforschung einen Hang zu haben, die Analyse moderner Grenzen unter historischen Maßgaben vorzunehmen, insofern als die Erforschung von Grenzen größtenteils erst mit dem 19. Jahrhundert einsetzt. Diese historische Kurzsichtigkeit hat die Anzahl sowohl der Grenztypen als auch der Theorien der Grenze, die die Grenzforschung entwickelte, willkürlich verringert. Dahingegen stellt die Critical Limology eine Methodologie dar, mit der eine weitaus größere Bandbreite an historischen und geografischen Grenzprozessen und -strukturen erfasst werden kann.

Die Fehleinschätzung, dass Grenzen menschliche Mobilität zur Gänze kontrollieren und das Eindringen unerwünschter Personen vollständig unterbinden können, ist heute Kern einer zunehmenden Zahl an menschlichen Tragödien (vgl. Brown 2010). Dieser Beitrag soll eine Einführung in einen an Bewegungen orientierten methodologischen Ansatz geben, der Grenzen nicht mehr als statische Werkzeuge des Separierens und Blockierens, sondern als historische Regime des Grenzziehens erforscht. Ich werde hier jedoch nicht den Raum haben, die empirischen und historischen Analysen zu vertiefen, die ich schon an anderer Stelle veröffentlicht habe (vgl. Nail 2016). Ziel dieses Beitrages ist es lediglich, einige Werkzeuge vorzustellen, die andere Forschende für ihre eigenen Zwecke als hilfreich empfinden könnten, damit Grenzforschung über ein breiteres Spektrum an Grenzstabilisierungsprozessen, geografischen Gegebenheiten und historischen Perioden hinweg analysiert werden kann.

\section{Kritische Methodologie}

Das erste wichtige Problem, mit dem sich Critical Limology beschäftigt, ist die Herausforderung, aus der Vielzahl von Ansätzen der Grenzforschung eine gemeinsame Theorie oder Methodologie der Grenztheorie zu entwickeln. Corey Johnson und Reece Jones (2011, S. 61) stellen fest: „the expansive understanding of borders and boundaries in recent scholarship has enriched border studies, but it has also obscured what a border is." Wenn Étienne Balibars (1998, S. 216-229) Behauptung „borders are everywhere“ zutrifft, dann sind sie ebenso gut nirgendwo. Und Linn Axelsson (2013, S. 325) merkt an: „we should be careful not to call everything a border. [..] the potential loss of analytical clarity if the border concept is used too broadly has been noted." Deshalb besteht ein bedeutendes methodologisches Problem 
für eine Theorie der Grenze darin, wie ein Konzept von Grenze entwickelt werden könnte, das sich nicht nur auf geografische Grenzen bezieht, sondern ihre vielfältigen Ausprägungen berücksichtigt. David Newman (2003, S. 124) beobachtet:

„What is sorely lacking is a solid theoretical base that will allow us to understand the boundary phenomena as it takes place within different social and spatial dimensions. A theory which will enable us to understand the process of ,bounding' and ,bordering' rather than simply the compartmentalized outcome of the various social and political processes."

Mit anderen Worten, was wir nach Ansicht Newmans brauchen, ist eine Theorie der Grenze, die den ursächlichen Prozess und nicht ein abgeleitetes sekundäres soziales Produkt in den Blick nimmt.

Nicht jede* $r$ stimmt jedoch darin überein, dass eine solche „solide theoretische Basis“ erreichbar oder gar erstrebenswert ist. Anssi Paasi (Johnson et al. 2011, S. 62) schreibt:

"A general border theory seems unattainable, and even undesirable, for two reasons. First, individual state borders are historically contingent and characterized by contextual features and power relations. There can hardly be one grand theory that would be valid for all borders. Such a theory is not problematic because the borders are unique but rather because of the complexity of borders and bordering."

Tatsächlich ist jede einzelne Grenze in der Geschichte empirisch einzigartig und beruht auf einer komplexen Gemengelage unterschiedlicher Machttypen. Vielleicht hat das explosionsartige Aufkommen neuer Grenztheorien in den letzten zehn Jahren nicht zur Entstehung einer neuen catch-all theory beigetragen, weil eine solche Theorie, wie Paasi feststellt (ebd., S. 62), bar jedes empirischen Gehaltes wäre, durch den sich jede einzelne spezifische Grenze auszeichnet, und damit sich selbst überflüssig machen würde, weil sie auf nichts mehr anwendbar wäre. Andererseits hat sich vielleicht der aktuelle Wunsch nach einer solchen Theorie der Grenze gerade aufgrund der zunehmenden Enttäuschung darüber ergeben, dass es den einzelnen empirischen Studien zu spezifischen Grenzen an weiterreichenden Implikationen, Konzepten oder Bezugsrahmen außerhalb ihrer eigenen beschränkten Reichweite mangelt.

Ohne einen solchen übertragbaren konzeptuellen Bezugsrahmen beginnt die empirische Erforschung der jeweiligen Grenze in all ihrer historischen Einzigartigkeit mit jeder Analyse wieder bei null, ohne Konsequenzen für zukünftige Untersuchungen oder andere Disziplinen außerhalb der Geografie zu haben. Diesem Empirismus ist es geschuldet, dass beispielsweise diejenigen, die die Trennung des Territoriums zwischen den Vereinigten Staaten und Mexiko untersuchen, eine völlig andere Grenze im Blick haben als diejenigen, die die juristischen Grenzen der Einwanderungsbehörden innerhalb der Vereinigten Staaten beleuchten. Die Idee, dass die Einwanderungsbestimmungen (also die juristische Grenze) und die Grenzkontrolle (als territoriale Grenze) absolut nichts miteinander zu tun haben, ist jedoch absurd, insbesondere nach ihrer politischen Zusammenführung unter dem Dach der US-Behörde Homeland Security.

Vor diesem Hintergrund möchte ich die Debatte zwischen den ,Sammel-'Theorien und der ,empirischen` Erforschung um eine alternative Herangehensweise bereichern. Bevor ich dazu komme, ist es aber wichtig, zwei Punkte zu klären. Erstens ist es nicht die Absicht einer ,Theorie‘ der Grenze, jedes einzelne Detail des empirischen Phänomens der Grenze zu beschreiben oder vorherzusagen: Eine Theorie der Grenze zielt vielmehr darauf ab, die dinghaften Voraus- 
setzungen oder die vielschichtigen Beziehungen darzustellen, innerhalb derer empirische Grenzen entstehen. Deshalb hat die Theorie der Grenze sowohl die vielfältigen Beziehungsgeflechte zum Gegenstand, die viele Grenzen gemeinsam haben, als auch die spezifischen Grenzen, die diese Beziehungen hervorbringen. Die Theorie der Grenze beleuchtet die Beziehungsgeflechte nicht außerhalb der kleinteiligen und empirischen geografischen Gegebenheiten, sondern in ihrer übergreifenden Qualität.

Zweitens erübrigen sich durch eine Theorie der Grenze und die Erforschung der allgemeinen Muster ihrer wiederkehrenden Bewegungen die empirischen Untersuchungen einzelner Grenzen keineswegs. Im Gegenteil, die empirische Abbildung der Gegebenheiten ist genau das, was allgemeinere Erkenntnisse im Hinblick auf bestimmte wiederkehrende Beziehungsgeflechte oder konzeptuelle Grenzregime ermöglicht. Darüber hinaus können sowohl empirische als auch konzeptuelle Arbeiten von dem Wissen über einige der grundlegenden wiederkehrenden historischen Strukturen oder Muster zirkulärer Bewegungen enorm profitieren. Eine Theorie der Grenze zeichnet sich also nicht durch die empirische Beschreibung jeder einzelnen Grenze aus. Critical Limology ist eine Theorie der Grenze, die sich zur empirischen Erforschung von Grenzen in ihrer Vielschichtigkeit und Neuartigkeit vollständig immanent und gegenläufig verhält. Dennoch wird die empirische Forschung in hohem Maße von einer breiteren theoretischen Basis profitieren und eine Einordnung der unterschiedlichen Grenzregime über die gesamte Geschichte und über die Disziplinen hinweg ermöglichen.

\subsection{Was ist kritisch an der Critical Limology?}

In der philosophischen Tradition bedeutet Kritik nicht das Aufspüren von Fehlern und Schwächen - es bezeichnet vielmehr den Verstehensprozess der Bedingungen bestimmter Sachverhalte. Critical Limology ist deshalb kritisch in dem Sinne, dass sie die materialen Bedingungen von Mobilität und wiederkehrenden Bewegungen erforscht, die für historische Grenztechnologien typisch sind. Kritik in dem Sinne, in dem ich sie verstehe, ist deshalb weder universell noch partikulär. Sie trägt dazu bei, eine hilfreiche Methodologie zu entwickeln, um diese Spannung, die den Forschungsgegenstand Grenze charakterisiert, aufzulösen.

Der Konflikt zwischen einer grand theory einerseits und dem wissenschaftlichen Empirismus andererseits, der die letzten Jahre der Grenzforschung geprägt hat, ist in der Philosophie keineswegs neu. Im achtzehnten Jahrhundert formulierte Immanuel Kant in der Kritik der reinen Vernunft (1787/1974) eine ähnliche Polemik zum Verhältnis zwischen Metaphysik und Empirismus. Einerseits, so Kant, sei die „Metaphysik ein[e] ganz isoliert[e] spekulativ[e] Vernunfterkenntnis, die sich gänzlich über Erfahrungsbelehrung erhebt“ (ebd., S. 24). Mit anderen Worten, das Wissen darüber, wie die Welt beschaffen ist, kann keine Begründung in unserer Erfahrung haben und damit auch weder Anwendung finden noch verifiziert werden. Andererseits „nahm man [bisher] an, alle unsere Erkenntnis müsse sich nach den Gegenständen richten; aber alle Versuche über sie a priori etwas durch Begriffe auszumachen, wodurch unsere Erkenntnisse erweitert würden, gingen unter dieser Voraussetzung zu nichte“ (ebd., S. 25). Das heißt, die empirischen Wissenschaften überfrachten uns mit detaillierten Informationen, verraten uns aber absolut nichts über die allgemeineren (A-priori-)Bedingungen des Wissens, unter denen diese Informationen uns als solches erscheinen. Kant löst dieses Problem, indem er es umkehrt, so wie Kopernikus es getan hat. Wir sollten stattdessen annehmen, so Kant, „die 
Gegenstände müssen sich nach unserer Erkenntnis richten“, so wie Kopernikus sich entschied, es sei besser, „wenn er den Zuschauer sich drehen und dagegen die Sterne in Ruhe ließ“ (ebd., S. 25). Mit anderen Worten, Kant schlug vor, stattdessen Regeln zu identifizieren, die „ich in mir, noch ehe mir Gegenstände gegeben werden, mithin a priori voraussetzen muss, welche in Begriffen a priori ausgedrückt wird, nach denen sich also alle Gegenstände der Erfahrung nothwendig richten und mit ihnen übereinstimmen müssen“ (ebd., S. 26). Kant bezeichnet diese philosophische Suche nach den Bedingungen möglicher Erfahrung als transzendentalen Idealismus oder als Kritik. Auf diese Weise umgeht er sowohl das Problem der grand theory (Metaphysik) als auch das des wissenschaftlichen Empirismus.

Dieser Einsicht folgend, schlage ich meine eigene Methodologie vor, um eine Theorie der Grenze zu entwickeln. Mein Ansatz stellt keine grand theory (Metaphysik) der Grenze selbst dar, die versucht, alle Grenzen im Voraus zu erklären und damit empirische Studien überflüssig zu machen. Ebenso wenig ist sie eine rein empirische Wissenschaft der Grenze wie die, die von frühen Grenzgeografen wie Jacques Ancel, Richard Hartshorn, Ewald Banse, Lord Curzon, Charles Fawcett und Thomas Holdich entwickelt wurde. Das Ziel der Theorie der Grenze in diesem Beitrag ist es, wie David Newman (2003, S. 134) klarstellt „[to develop] a solid theoretical base that will allow us to understand the boundary phenomenon as it takes place within different social and spatial dimensions".

Critical Limology beinhaltet die Erforschung materialer sozialer und kultureller Grenzen, z.B. territorialer, politischer, juristischer und wirtschaftlicher Art. Sie gründet sich nicht nur auf spekulative oder metaphysische Separierung oder Begrenzungen, sondern auf strikte historische und empirisch erfahrbare Grenztechnologien wie Zäune, Mauern, Zellen und Grenzübergänge, die allgemein und über lange Zeiträume hinweg verstanden werden. ${ }^{1}$

Weil Grenzen dinghafte und technologische Praktiken sind, müssen Grenzen gemacht werden. Wenn Grenzen gemachte Gebilde sind, dann können sie auch auf andere Weise gestaltet werden. Deshalb sind Gegenbewegungen des Widerstandes ein konstitutiver Bestandteil von Grenzprozessen - insofern, als soziale Mobilität die Entstehung von Grenzen ursächlich bedingt. Aus diesem Grund kann soziale Mobilität niemals vollständig oder endgültig durch irgendein Geflecht von Grenzregimen erfasst werden. Alle Grenzen sind durchlässig, weil sie von und durch einen Prozess der Mobilität oder des sozialen Verkehrs erschaffen und dann in Form von Grenzregimen stabilisiert werden. Eine wichtige Konsequenz dieses kinetischen Ansatzes ist, dass Grenzen jeglicher Art ständigen Kontroversen und Veränderungen ausgesetzt waren, weil im Laufe der Geschichte viele unterschiedliche Typen des Widerstandes gegen Grenzpraktiken entstanden und wieder verschwunden sind. Ich appelliere deshalb an die Leser*innen, die Theorie und Geschichte der Grenze in diesem Beitrag und in Theory of the Border (Nail 2016) um den Aspekt der Migration zu ergänzen, den ich ebenso wie andere (vgl. Simpson 2014; Vollmer/Düvell in diesem Band) an früherer Stelle entwickelt habe.

1 Darüber hinaus wird in diesem Beitrag weder eine vollständige Theorie des Widerstands gegen Grenzen noch eine Typologie der politischen Subjekte, die sie bekämpft haben, entwickelt. Dies ist aus mehreren Gründen der Fall: zum einen, weil eine solche Theorie schon an anderer Stelle ausführlich in The Figure of the Migrant (Nail 2015 ) entwickelt wurde und es redundant wäre, sie hier noch einmal darzulegen. Zum anderen finden sie in diesem Beitrag keine Erwähnung, weil es erstens Ziel dieses Beitrags ist, die historischen Bedingungen bestimmter prävalenter Grenzregime zu diagnostizieren und zweitens Anti-Grenz-Bewegungen keine Grenzen sind. Theorie und Geschichte des politischen Widerstandes erfordern den Einsatz geringfügig anderer theoretischer Werkzeuge als derjenigen, die erforderlich sind, um die Aufrechterhaltung von Grenzen zu verstehen. Ein einzelner Beitrag in einem Buch kann nicht alles leisten. Ich bitte deshalb die Leser*innen, die willkürliche Unterscheidung zwischen Gegenbewegung und Grenzmacht zu entschuldigen. 
Eine Grenze ist nicht einfach nur eine empirisch erfahrbare Technologie, gegen die man Widerstand leisten kann oder auch nicht; sie ist darüber hinaus Teil viel größerer historischer Bewegungsmuster, die die empirisch erfahrbaren Grenztechnologien organisieren. Was ich als ein Grenzregime bezeichne, transzendiert nicht die dinghaften Technologien, die es konstituieren. Das Grenzregime ist vielmehr ihre Bedingung, Struktur oder Beziehung, nicht ihre Ursache, und ist analog zu der Art und Weise, wie die dinghaften Grenztechnologien selbst zusammengestellt werden, Veränderungen unterworfen. Deshalb ist die Critical Limology insofern materialistisch, als sie Grenzen als Regime realer performativer Techniken betrachtet und nicht in erster Linie als Ideen, als Ideologien des Separierens oder als Wissen, das unabhängig von sozialen und materiellen Bedingungen auftaucht: Es gibt, wie der Dichter William Carlos Williams (1946/1995, S. 6) schreibt, „keine Ideen außer in [den] Dingen“.

Critical Limology folgt deshalb grob der kritischen Tradition der Philosophie, wie im Folgenden beschrieben. Es gibt immanente Bedingungen oder Muster wiederkehrender Bewegungen, unter denen empirisch erfahrbare Grenzen entstehen, aber hierbei handelt es sind nicht, wie bei Kant, um mögliche, sondern im Gegenteil um reale Bedingungen, die zutiefst sozial, material und historisch bedingt sind. Mit anderen Worten, es gibt nicht eine Reihe universeller A-prioriKonzepte, die die Existenz jeder Grenze und aller Grenzen erklären. Es gibt jedoch zahlreiche unterschiedliche historische Muster sozialer wiederkehrender Bewegungen, auf deren Grundlage die meisten prävalenten sozialen Grenzen funktioniert haben. Die Art und Weise, wie diese dem Bewusstsein erscheinen, ist nicht idealistisch im Sinne der transzendentalen Logik nach Kant, aber sie ist ebenso wenig ausschließlich empirisch erfahrbar, weil es sich nicht um rein passive mechanistische Dinge oder Objekte in der Welt handelt. Sie sind in transzendentalem Sinne empirisch, historisch oder materiell insofern erfahrbar, als sie aktiv die realen Strukturen sozialer Bewegung und Separierung leisten und ununterbrochen umgestalten. Grenzen sind keine ,Dinge‘, sie stellen Prozesse oder kinetische Muster sozialer Bewegung dar. Critical Limology erforscht deshalb die realen materialen und immanenten Bedingungen sozialer Mobilität. Sie ist kritisch, nicht weil sie Grenzen schlicht als falsch empfindet (es gibt viele Aspekte der Grenze, die man als falsch empfinden kann), sondern weil sie an die tieferen historischen und materialen Strukturen heranreicht, die durch spezifische Grenztechnologien und ihre jeweilige Kombination erschaffen werden. ${ }^{2}$

\section{Grenze als Prozess konstanter Zirkulation}

In ihrer allgemeinsten Definition ist Grenze ein kinetischer Prozess der Verzweigung (bifurcation) und der wiederkehrenden Bewegung (circulation). Ich verstehe diese Definition nicht als abstraktes Konzept, sondern als etwas, das aus der historischen und empirischen Erforschung von Grenzen, die in Theory of the Border (Nail 2016) geleistet wird, abgeleitet werden kann. Seit diese Muster und Technologien der Grenzziehung in der Geschichte sichtbar und zum Forschungsgegenstand wurden, ist nachvollziehbar, wie sie nicht einfach nur die Vergangenheit überdauert haben, sondern auch in der Gegenwart fortbestehen. Hier fehlt es an Raum, um alle Bewegungsmuster zu beschreiben (bestimmt durch Zentripetal- und Zentrifugalkraft, Spannkraft und Elastizität), die sich auf die vier vor allem im Westen vorherrschenden Grenztechnologien (Zäune, Mauern, Zellen und Grenzübergänge) zurückführen lassen. Ich möchte

2 Kritische Grenzforschung ist außerdem kritisch insofern, als gezeigt werden soll, dass die Geschichte der vorherrschenden Grenzregime keine notwendige ist. Weil die Bedingungen nicht wesentlich oder universell sind - sondern historisch und beweglich -, könnten die Betongrenzen auch anders aussehen als heute. 
jedoch gern versuchen und anhand des Beispiels der US-amerikanisch-mexikanischen Grenze aufzeigen, inwiefern Grenzen weniger dazu da sind, Mobilität zu unterbinden als vielmehr dazu, diese in ständiger Bewegung zu halten.

Die Grenze zwischen den USA und Mexiko zeichnet sich durch Muster wiederkehrender Bewegungen aus, in denen wir mindestens drei Kreisläufe erkennen können. Der erste ist der unmittelbare Grenz-Kreislauf (border circuit), der seinerseits aus drei Bewegungen besteht: (1) Migrierende überqueren (cross) die Grenze. Die Grenze agiert dabei gleichzeitig als Knotenpunkt, an der diese Ströme zusammenlaufen. Sie wirkt wie ein Sieb oder Filter, weil sie dem Kapital und der globalen Elite erlaubt, sich frei zu bewegen, während sie gleichzeitig die Armen und Bedürftigen wie ein Joch abfängt und festsetzt. (2) Ein Strom von Migrierenden überquert die Grenze, ob legal oder illegal, und falls die Migrierenden ihren Status verloren haben, werden sie von den Betreiber*innen der Grenz-Knotenpunkte - den Grenzschutzbeamtinnen festgesetzt (apprehended). Ebenso gut kann der Strom der Migrierenden die Grenze passieren und später weit davon entfernt wieder aufgegriffen werden - Raum und Nähe sind nicht die ausschlaggebenden Aspekte. Vielmehr ist es die militarisierte, legalisierte und politische Grenze, die den kriminellen Akt erst erschafft. Sie deutet die Mobilität der Migrierenden um und bezeichnet sie als illegal. Die Immigrationsbehörden werden zu Grenzbehörden, egal wo sie eingreifen. (3) Der festgesetzte Strom der Migrierenden wird der Exekutive überstellt und dann abgeschoben (deportation), d.h. wieder über die Grenze zurückgeschickt. Dort werden die abgeschobenen Migrierenden wieder freigelassen und der Kreislauf beginnt von vorn. Der Grenzverkehr setzt sich also aus Übertritt, Festsetzen, Abschiebung und erneutem Übertritt der Grenze zusammen (cross, apprehend, deport, cross: C-A-D-C). Jeder Zyklus in diesem Kreislauf generiert Geldmittel, Macht und Prestige auf Seiten der Einwanderungsbehörde und rechtfertigt damit Reproduktion und Ausbau der Behörde. Das Unterbrechen oder Blockieren dieses Kreislaufs würde das gesamte Regime zerstören.

Der zweite Kreislauf ist der Internierungs-Kreislauf (detention circuit), der mit dem Übertritt der Grenze durch die Migrierenden beginnen kann oder auch den Beginn einer zusätzlichen Schaltstelle des Grenzkreislaufs markiert, nachdem die Migrierenden festgesetzt wurden. Der Internierungs-Kreislauf besteht ebenfalls aus drei grundlegenden Teilen: (1) Migrierende übertreten die Grenze und werden festgesetzt. (2) Statt unverzüglich ausgewiesen zu werden, werden sie in einen weiteren Knotenpunkt überführt - ein Gefängnis, ein Internierungslager oder eine zentrale Aufnahmestelle. Der Strom der Migrierenden wird somit in das Aufnahmelager ausgedehnt. Das Aufnahmelager macht sich als Knotenpunkt wiederum die Mobilität der Migrierenden nutzbar bzw. entzieht ihnen diese in Form ihrer Arbeit und aufgrund ihrer Unterbringung und des Konsums, den die Internierung selbst mit sich bringt: benötigt werden Nahrungsmittel, Wasser, Kleidung, medizinische Versorgung und so weiter (dieser Konsum garantiert privatwirtschaftliche Gewinne, die massiv durch die Regierung subventioniert werden). In den Vereinigten Staaten sind zum Beispiel „18.690 der 32.000 Immigranten, die sich in Gewahrsam befinden, nicht strafrechtlich verurteilt worden. Mehr als 400 der Personen ohne strafrechtlich relevante Vergehen sind seit mindestens einem Jahr in Gewahrsam “ (DHS 2009). (3) Sobald das maximale Ausmaß an Mobilität aus dem Strom der Migrierenden extrahiert wurde - manchmal nach langen Jahren der Internierung - werden die Migrierenden ausgewiesen. Sobald sie abgeschoben worden sind, kann der Kreislauf wieder von vorn beginnen oder aber wie eine Schaltstelle in den nächsten Kreislauf agieren. Der Internierungs-Kreislauf 
lautet also Festsetzen, Internierung, Abschiebung, Internierung (apprehend, detain, deport, apprehend: A-DT-D-A).

Der dritte Kreislauf ist der Arbeitsmarkt-Kreislauf (labor circuit; vgl. auch Mezzadra/Neilson in diesem Band). Auch dieser besteht aus drei Teilen: (1) Ein Strom von Migrierenden übertritt die Grenze entweder legal oder illegal. Dies kann sowohl nach der Internierung und anschließender Abschiebung (DT-D) oder nach dem Festsetzen mit anschließender Abschiebung (A-D) oder auch nach dem ursprünglichen Grenzübertritt (C) geschehen. (2) Die Migrierenden werden dann in einen Arbeitsknotenpunkt überführt, der darauf abzielt, den Migrierenden so viel Mobilität wie möglich abzuringen. Arbeitgeber und Wirtschaft können mehr abschöpfen, wenn die Migrierenden keinen Status haben, als wenn Migrierende legal eingereist sind weil der Zugang zu Gewerkschaften verhindert wird, durch eine drohende Abschiebung oder aufgrund niedrigerer Löhne und gefährlicherer Arbeitsbedingungen. In diesem Falle sind die Kapitalist*innen die Fahrer*innen des Arbeitsmittels, das, ohne eigenständige Bewegung, von den Migrierenden bewegt wird. Die Schubkraft (Bewegung), die aus der Arbeitskraft der Migrierenden resultiert, zieht das Fahrzeug, das sich unter dem Joch der Kapitalist*innen befindet. (3) Vom Arbeitsmarkt-Knotenpunkt können die Migrierenden unter Umständen wieder über die Grenze zurückkehren, dann wieder zur Arbeit kommen und so weiter, bis einer der anderen Kreisläufe durch Festsetzen, Internierung oder Abschiebung einsetzt.

Der Arbeitsmarkt-Kreislauf zielt darauf ab, die Extraktion der Mobilität aus dem Strom der Migrierenden ins Unendliche auszudehnen und diese in die vielfältigen Knotenpunkte der Wirtschaft einzubringen. Statt auf das geringere Maß im Aufnahmelager zurückreguliert zu werden, wird der Strom in den größten der drei Kreisläufe ausgeweitet: den unermesslich großen Kreislauf des Arbeitsmarktes. Das Ziel dieses Kreislaufs ist es, eine Wirtschaftsform auf Grundlage der entmachteten Migrierenden-Arbeitskraft zu reproduzieren, die ein Gegengewicht zur durch Mitspracherechte und höhere Löhne gekennzeichneten Arbeitsleistung der Personen mit staatsbürgerlichen Rechten schafft. Der Arbeitsmarktkreislauf besteht also aus: Übertritt, Arbeit, Übertritt, Arbeit (C-W-C-W ... D-C).

Die Bewegungen innerhalb der und zwischen diesen Kreisläufen stellen die wiederkehrenden Bewegungen an der Grenze dar. Die Grenze ist weder ein statisches Objekt noch sorgt sie dafür, die Mobilität der Menschen zu unterbinden, noch ist sie rein ideologischer Natur. Vielmehr handelt es sich um ein aktives materiales System, das in der Lage ist, sich selbst jenseits der Kontrolle eines einzelnen Individuums zu reproduzieren.

Dies sind nur drei Kreisläufe eines Typus wiederkehrender Bewegungen. Grenzen sind jedoch noch weitaus vielschichtiger. Grenzen und soziale Mobilität sind durch Ströme, Knotenpunkte und wiederkehrende Bewegungen definiert.

\section{Zur Geschichte der Grenze}

Das zweite wichtige Problem, das die Critical Limology ins Visier nimmt, ist die Tatsache, dass die Geschichte der Grenze sich hauptsächlich auf Grenzen zwischen Nationalstaaten konzentriert hat (vgl. Diener/Hagen 2012). In älteren Forschungsarbeiten und im öffentlichen Diskurs sind Grenzen vor allem als äußere territoriale Begrenzungen von Staaten definiert und werden mit abstrakten Linien und klar gekennzeichneten Grenzverläufen identifiziert. Dies ist weder in Bezug auf vormoderne Grenzen korrekt noch trifft es auf moderne Grenzen zu. Die Grenze als sozialer Prozess wiederkehrender Bewegungen und Separierungen lässt sich nicht 
auf die Staatsgewalt und erst recht nicht auf eine abstrakte Linie, die über die Erde verläuft, reduzieren. Vielmehr stellt sie einen Prozess der Verzweigung dar, den Staaten sich zunutze machen wollen, der sich ihnen jedoch immer wieder entzieht. Grenzen sind nicht nur viel älter als Staaten, weil Menschen schon Tausende von Jahren vor der Existenz von Staaten Grenzen gezogen haben. Das Phänomen der Grenze geht dem Staat auch in logischer Hinsicht voraus, insofern, als Grenze die technische Separierung repräsentiert, die erforderlich ist, um eine soziale Unterteilung vorzunehmen, die als Staat bezeichnet wird. Eine Geschichte der Grenze kann nicht auf die Geschichte von Staaten oder Mauern reduziert werden. Entsprechend gibt es eine vielgestaltige Geschichte der Grenze, deren Bedeutung durch den alleinigen Fokus auf Staatsgrenzen und auf die Geschichte der Neuzeit seit Beginn des 19. Jahrhunderts überschattet oder vollständig ignoriert wurde.

Critical Limology ermöglicht eine neuartige Geschichte, Theorie und Erforschung von Grenzen, weil sie die Muster der Mobilität erforscht (und nicht Staaten oder moderne Grenzen). Critical Limology ist eine Geschichte sozialer wiederkehrender Bewegungen, die auch Staaten als Produkte des Grenzentstehungsprozesses berücksichtigen. Statt Grenzforschung als Unterkategorie der Politikwissenschaft oder der Geografie zu verstehen, plädiere ich dafür, dass wir die Dinge genau andersherum betrachten sollten. Gesellschaft und Geografie sind Produkte des Prozesses der Grenzziehung.

Das bedeutet nicht, dass die Critical Limology eine neue universelle Geschichte anzubieten hätte. Das Gegenteil ist der Fall. Die Erforschung der Materialität von Techniken der Grenzziehung soll unsere Aufmerksamkeit wieder auf die Bedingtheiten einer nichtanthropozentrischen Geschichte der Regime zirkulärer Bewegungen richten, die mit menschlichen und nichtmenschlichen Akteuren gleichermaßen angefüllt sind. Critical Limology soll eine kinetische Geschichte sein, die aus den empirischen geschichtlichen Fakten diejenigen Konzepte herausfiltert, die die Entstehung einer kritischen Grenzforschung ermöglichen, die wiederum für die zeitgenössische Analyse von Nutzen ist, in der wir die ganze Geschichte der bis in die Gegenwart bestehenden Grenzen finden. Die Vergangenheit zu verstehen liefert den Schlüssel, um die Gegenwart zu interpretieren.

Ich muss zugeben, dass ich selbst die Critical Limology in der Theory of the Border (Nail 2016) ausschließlich auf die Geschichte des Westens und des Nahen Ostens angewendet habe. Aber es gibt keinen Grund, warum zukünftige Projekte nicht die Erforschung von Grenztechnologien und ihre Prozesse wiederkehrender Bewegungen weltweit in den Blick nehmen können. Eines der bedauerlichen Opfer, die zugunsten der historischen Breite der Theory of the Border gebracht werden mussten, resultierte in dem eingeschränkten geografischen Blickwinkel. Um mehrere prävalente Grenzregime möglichst exakt theoretisch darzustellen, musste ich die Studie auf ihre augenfälligsten Ausprägungen der westlichen Geschichte reduzieren. Diese Methode barg das Risiko, den Eindruck zu erwecken, dass dies die einzigen Manifestationen von Grenzregimen sein könnten, die einzigen, die von Bedeutung sind oder auch die einzig möglichen - nichts von alldem ist der Fall. Vielmehr wollte ich, indem ich mich auf die vorherrschenden Grenzregime konzentrierte, genau das Gegenteil aufzeigen: Weil diese Regime im historischen Kontext sichtbar werden und weder notwendig noch entwicklungsbedingt sind, können sie oder werden sie eventuell auch andere Ausprägungen annehmen als sie es derzeit tun oder es hätten tun können. Auf diese Weise birgt Critical Limology die Möglichkeit des Widerstandes gegen diese dominanten Regime, selbst wenn sie nicht alle wichtigen historischen 
Strategien des Grenzwiderstandes nacherzählen, die Thema meiner Publikation The Figure of the Migrant (Nail 2015) sind.

Es gibt drei wesentliche Gründe, eine Theorie der Grenze auf Grundlage einer kritischen und materialen Geschichte der Grenze zu erarbeiten. Erstens erlaubt uns diese Herangehensweise, die historischen Bedingungen zu konzeptualisieren, unter denen verschiedene Ausprägungen sozialer Grenztechnologien produziert worden sind. Dieser Ansatz vermeidet auch den Kant'schen Idealismus, der darauf abzielen würde, die ahistorischen Bedingungen sämtlicher Grenzen zu bestimmen. Leider gibt es eine Tendenz unter den Grenzforschenden, die Geschichte der Grenze erst ab dem 19. Jahrhundert zu schreiben, als die Grenzforschung damit begann, Grenzen als die äußeren territorialen Begrenzungen von Nationalstaaten zu beschreiben. Grenzen sind jedoch nicht erst im 19. Jahrhundert entstanden. Darüber hinaus lassen Konzepte nationaler oder militärischer Verteidigung nur wenige Rückschlüsse auf die ursprünglichen Trennlinien zu, auf die Nationalstaaten letztendlich zurückgehen. Die Geschichte der Grenze ist komplexer und trägt viele Namen. Grenze erscheint überall, wo physische Technologien sozialer Separierung und wiederkehrender Bewegung auffindbar sind. Es gibt also unterschiedliche Arten von Grenzen, die zu unterschiedlichen Zeitpunkten in der Geschichte und in Abhängigkeit von den sozialen Bedingungen und Bewegungen, die spezifisch für die Art ihrer Separierung sind, entstanden sind.

Zweitens sind die Theorie der Grenze und die Geschichte ihrer immanenten technischen Ausprägungen hilfreich, um derzeit bestehende Grenzen zu analysieren. Das ist möglich, weil die Geschichte der Grenze keinen linearen oder progressiven geschichtlichen Verlauf über unterschiedliche Epochen hinweg darstellt, sondern vielmehr eine Geschichte koexistierender und einander überlappender Muster sozialer Mobilität ist. Grenze ist nicht etwas, das plötzlich auftaucht und dann wieder verschwindet. Im Sinne physischer Grenztechnologien haben Grenzen in der Regel die Eigenschaft, sich selbst mit Hilfe grundlegender technologischer Strukturen in Form von Zäunen, Mauern, Zellen und Grenzübergängen zu reproduzieren. Sie bestehen fort, verändern sich, wirken zusammen und koexistieren in neuen sozialen Kontexten und in Form neuer Materialien. Als Regime der Mobilität bestehen Grenzen auch in unterschiedlichem Ausmaß über geschichtliche Zeitläufte hinweg fort, entwickeln sich und verschmelzen miteinander. Um gegenwärtig existierende Grenzen zu verstehen und angemessen auf sie zu reagieren, müssen wir also auch das Entstehen und die Koexistenz aller Arten von historischen Grenzen und die Bedingungen, unter denen sie im Lauf der Geschichte entstanden sind, verstehen.

Im Gegensatz zu einem rein empirischen Ansatz trachtet die Critical Limology jedoch nicht danach, neue empirische Grenztechnologien vorherzusagen, sondern zielt darauf ab, die gegenläufigen Muster wiederkehrender Bewegungen an früheren und heutigen Grenzen zu verstehen. Häufig ist das, was die heutige Generation der Forschenden als ,neuartige“ Technologien und Formen der Grenzstabilisierung verstehen, nichts als eine Neukombination alter Regime und Technologien, die schon seit Hunderten oder sogar Tausenden von Jahren existieren. ${ }^{3}$ Eine kritische Grenzforschung, die die Geschichte der Grenztechnologien und die Muster wiederkehrender Bewegungen ernst nimmt, kann auch verhindern, dass Grenzforschende jedes Mal

3 Siehe Wendy Brown (2010): Ihr Argument lautet, dass Mauern als verzweifelte und gescheiterte Versuche angesehen werden können, mit zunehmenden Phänomenen der Auflösung von Grenzen umzugehen. Historisch betrachtet haben Mauern noch nie funktioniert. Es ist keinesfalls so, dass Mauern zu irgendeiner Zeit funktioniert hätten und heute aufgrund von Krankheiten, Migration, Schmuggel und grenzüberschreitenden Organisationen und so weiter nicht mehr funktionieren. Viele dieser Phänomene haben auch in der Geschichte in der einen oder anderen Form schon existiert. Mauern haben Menschen noch niemals davon abgehalten, irgendwo hinzugelangen. 
das Rad der Grenztheorie vermeintlich neu erfinden, wenn eine sogenannte ,neue` Technologie auftaucht.

Drittens stellt die kritische Analyse vergangener und heutiger Grenzregime die notwendigen Werkzeuge zur Verfügung, die notwendig sind, um die derzeit bestehenden Regime zu verändern. Wer versteht, wie eine Grenze funktioniert, kann effektivere taktische Interventionen vornehmen, um sie zu verändern oder abzuschaffen. Die kinetische These der Critical Limology beruht auf der Annahme, dass Grenzen keine universelle oder von geschichtlichen Gegebenheiten unabhängige soziale Notwendigkeit haben und deshalb die Möglichkeit in sich tragen, in Zukunft verändert oder abgeschafft zu werden - wie die Geschichte zeigt.

Und dennoch liefert Critical Limology keine metaphysisch normative Theorie dessen, was wir tun sollten, statt diese Art von Grenzen zu errichten. Diese Frage richtet sich vielmehr an uns alle - im weitesten Sinne des Wortes uns - und schließt die Migrierenden, die direkt von derartigen Regimen betroffen sind, mit ein (vgl. Nail 2015). Stattdessen liefert Critical Limology allein durch den Kontrast zu zuvor etablierten historischen Regimen die kinetische Analyse, durch die echte Alternativen erkennbar werden (vgl. ebd.). ${ }^{4}$

Die immanenten materialen und historischen ,Bedingungen“ der Critical Limology haben nichts mit Kausalität oder Notwendigkeit zu tun. Nur weil wir Grenzen in der Vergangenheit auf diese Weise gezogen haben, heißt das nicht, dass wir in Zukunft genauso verfahren müssen. Die Analyse zeitgenössischer Grenzen zielt nicht auf eine umfassende kausale Erklärung ab, sondern stellt eine deskriptive Analyse dar, die uns helfen soll, zu sehen, was tatsächlich hinter der Rhetorik steckt, dafür zu sorgen, dass ,unerwünschte Personen draußen bleiben'. Critical Limology setzt mit einer immanenten Analyse dessen ein, was für Grenzmuster geschaffen worden sind, und versucht zu zeigen, welche materialen und historischen Bedingungen diesen Mustern zugrunde liegen. Das Ziel lautet also nicht, die Ursachen aller Grenzen zu erklären, sondern aus der Perspektive dieser Verzweigungen ähnelnden Bewegungsstruktur bessere Beschreibungen der Bedingungen, Handlungskapazitäten und Verläufe ihrer historischen Entstehung und Koexistenz in der Gegenwart anzubieten.

Viele Theorien der Grenze behandeln die Grenze als etwas Statisches, über das sich Menschen hinwegbewegen - vergleichbar einer semi-permeablen Membran oder sogar einer Zone. ${ }^{5} \mathrm{Je}$ intensiver sich der Blick jedoch auf die materiale Handlungsfähigkeit der Grenze - als sich je nach geografischer, geologischer, topologischer, dem Verfall anheimgegebener oder einer Vielzahl anderer nichtmenschlicher Prozesse wandelnde Muster - richtet, desto mehr erkennen wir, dass die Grenze selbst durch einen konstanten Zustand der Mobilität definiert ist, der nicht ausschließlich menschlicher Kontrolle unterliegt. Eine Grenze wird nicht nur durch menschliche Eingriffe, sondern auch durch das Wettergeschehen, durch geologische Gegebenheiten, Tiervorkommen, ökologische Bedingungen und den materiellen Verfall der Grenzanlagen selbst unablässig reproduziert. Darüber hinaus betrachten die meisten Theorien der Grenze diese als das Produkt von Staaten, Gesetzen und gesellschaftlichen Kräfte. Aus der Perspektive der Critical Limology hingegen ist es der dinghafte Prozess der Grenzziehung selbst, der in der historischen Betrachtung dem Erstarken von Staaten und Gesellschaften (die in der Folge teilweise die Grenze reproduzieren können) vorausgeht. Sobald politische und nationale Staaten entstanden sind, setzen sie lediglich die Reproduktion der Grenzen

4 Die kinepolitische Struktur dieser Alternativen wird beispielhaft, aber sicherlich nicht ausführlich in The Figure of the Migrant (Nail 2015) dargestellt.

5 Eine Beschreibung und Kritik dieses Ansatzes findet sich in Nyers (2012, S. 2-6). 
fort, die ihre Entstehung erst möglich gemacht haben. Diese setzen geologische Materialien, ökologische Systeme, extraktive Technologien und das Vorhandensein bestimmter natürlich vorkommender Materialien wie Holz, Gestein, Ziegel, Draht und Beton voraus. Statt also Grenzen als zum einen statische, zum anderen in der Rolle von Statisten gefangene Objekte zu betrachten, schlägt die Critical Limology eine neue materiale und kinetische Theorie der Grenze vor, die die Grenze selbst als Teil zirkulärer Prozesse ernst nimmt (vgl. Nail 2016).

\section{Grenzen als Prozesse der Expansion durch Enteignung}

Drittens sorgt die Critical Limology dafür, dass die Frage der Grenztheorie nicht mehr Was ist eine Grenze? lautet, sondern Was macht eine Grenze? oder Wie bewegt sich eine Grenze?. Genauer ausgedrückt, möchte ich gern argumentieren, dass Grenzen zirkulierende Prozesse darstellen, in die sich bestimmte soziale Bewegungen ausdehnen, indem sie andere in einen metastabilen Zustand versetzen. Dies ist eines der wenigen Dinge, die jede wichtige historische Grenze bislang getan hat.

Meine These hier lautet, dass wir Grenzen nicht mehr als Objekte, sondern vielmehr als weitläufige Prozesse oder komplexe Systeme denken sollten, nicht nur im Sinne wirtschaftlicher Enteignung und Aneignung, sondern eher im Sinne sozialer Aneignung. Ich nenne diesen Ansatz Expansion durch Enteignung (vgl. Schetter/Müller-Koné in diesem Band). Der Prozess, Menschen ihres sozialen Status zu berauben (expulsion), um eine gegebene Form sozialer Mobilität weiterzuentwickeln oder zu beschleunigen (expansion), ist keineswegs auf das kapitalistische Regime sozialer Mobilität beschränkt (Marx 1967/1986). Wir erkennen den gleichen sozialen Prozess in frühen menschlichen Gesellschaften, deren fortschreitende Kultivierung von Land und Tieren (territorial expansion) mit der physischen Technologie des Einzäunens auch einen Teil der menschlichen Bevölkerung ausgeschlossen hat (territorial dispossession). Dies trifft sowohl auf Sammler- und Jäger-Gesellschaften zu, deren Territorien in landwirtschaftliche Fläche verwandelt wurden, als auch auf Landwirte, die eine zu intensive Landwirtschaft betrieben haben und denen ab einem bestimmten Zeitpunkt kein urbares Land mehr zur Verfügung stand. Deshalb ist die soziale Enteignung in zweierlei Hinsicht Bedingung sozialer Expansion: Es handelt sich um eine interne Bedingung, die vorsieht, einen Teil der Bevölkerung zu enteignen, wenn gewisse interne Grenzen (wie zum Beispiel die Aufnahmefähigkeit eines bestehenden Territoriums) überschritten worden sind, und es handelt sich um eine äußere Bedingung, die zulässt, einen Teil der Bevölkerung außerhalb dieser Grenzen zu entfernen, wenn das Territorium sich in das Land anderer Gruppen (Jäger und Sammler) ausdehnen kann. Im historischen Rückblick war die territoriale Expansion nur möglich, wenn dieser Teil der Bevölkerung vertrieben und gezwungen wurde, als migrierende Nomaden in die umliegenden Berge oder Wüsten zu ziehen.

Später vollzog sich genau die gleiche Logik in der antiken Welt, deren vorherrschende politische Form, der Staat, nicht ohne die physische Technologie der Grenzbefestigung, die nicht nur Feinde davon abhielt, von außen einzudringen, sondern auch dazu diente, eine beträchtliche Anzahl an Barbaren aus den gebirgigen Gegenden des Mittleren Ostens und des Mittelmeerraumes (durch politische Enteignung) als Sklaven gefangen zu halten, möglich gewesen wäre. Die sozialen Bedingungen der Expansion einer wachsenden politischen Ordnung, zu denen auch die Kriegsführung, der Kolonialismus und öffentliche Bauarbeiten (Infrastrukturmaßnahmen) gehörten, entsprachen exakt der Enteignung einer Bevölkerung von Barbaren, die mittels 
politischer Machtstrukturen sowohl ein- als auch ausgesperrt werden mussten. Diese Technik taucht in der Geschichte wieder und wieder auf, wie ich in meinen Arbeiten zu zeigen versuche.

Der Prozess, eine Enteignung oder soziale Abwertung zu betreiben, um eine soziale Expansion zu bewirken, beschränkt sich nicht nur auf territoriale oder juristische Aspekte. Expansion ist keineswegs nur wirtschaftlicher Natur, wie wir es von Marx kennen. Enteignung bedeutet nicht einfach, Menschen von ihrem Land zu vertreiben, auch wenn dies in vielen Fällen dazugehört. Es bedeutet auch, dass Menschen ihrer politischen Rechte beraubt werden, indem sie aus der Stadt ausgeschlossen werden, bestimmte Gruppen durch die zellulären Techniken des Einsperrens und Einkerkerns zu kriminalisieren oder ihren Zugang zur Arbeit durch Identifikations- und Grenzübergangstechniken zu beschränken. Enteignung ist das Ausmaß, in dem ein politisches Subjekt eines bestimmten Status in der sozialen Ordnung beraubt wird. Entsprechend weiten Gesellschaften ihre Macht also in mehrfacher Hinsicht aus: durch territoriale Aneignung, durch politische Macht, unter Zuhilfenahme der Rechtsordnung und mittels wirtschaftlicher Profite. Die marxistische Theorie ursprünglicher Akkumulation und das Konzept der kinetischen Expansion durch Enteignung haben gemeinsam, dass dem Großteil bedeutender Expansionen sozialer kinetischer Macht eine frühere oder primitive Gewalt kinetischer sozialer Enteignung vorausging. Grenzregime bestehen aus den kinetischen Beziehungen und dinghaften Technologien, die diese Enteignung direkt vollziehen. Marx’ Konzept der ursprünglichen Akkumulation ist nicht mehr als ein historischer Moment einer allgemeineren Grenzlogik, die das Entstehen und die Reproduktion von Gesellschaften begleitet.

Zusammengefasst liegt den physischen kinetischen Bedingungen für die Expansion von Gesellschaften der Einsatz zirkulärer Systeme (Zäune, Mauern, Zellen, Grenzübergänge) zugrunde, mit deren Hilfe ein Regime marginalisierter territorialer, politischer, legaler und wirtschaftlicher Minderheiten geschaffen wird, die an anderer Stelle leichter wieder in den Kreislauf eingebracht werden können, sofern dies erforderlich ist. So wie die vagabundierende Minderheit durch Einsperren enteignet und in ein wirtschaftliches Proletariat verwandelt wird, so hat jedes herrschende soziale System seine eigene Struktur der Expansion durch Enteignung. Und genau darum geht es der Critical Limology.

\section{Fazit}

Wir leben in einer Welt der Grenzen. Territoriale, politische, juristische und wirtschaftliche Grenzen aller Art definieren im 21. Jahrhundert buchstäblich jeden Aspekt des sozialen Lebens. Obwohl die Globalisierung und die zunehmende Notwendigkeit globaler Mobilität gefeiert werden, gibt es heute mehr Arten von Grenzen als jemals zuvor in der Geschichte. In den letzten zwanzig Jahren und insbesondere seit 9/11 sind weltweit Hunderte neuer Grenzen entstanden: kilometerlange neue Stacheldrahtzäune, tonnenweise neue Betonsicherheitsmauern, zahllose Asylbewerberaufnahmelager in Übersee, biometrische Passdatenbanken sowie Sicherheitskontrollpunkte in Schulen, an Flughäfen und entlang unzähliger Straßen in aller Welt.

Moderne soziale Mobilität wird heute überall zergliedert (vgl. Schindler in diesem Band). Sie wird in territorialer Hinsicht durch Zäune um unsere Häuser, Institutionen und Länder eingehegt. Sie wird politisch durch das Militär, durch Grenzmauern und Eingangsportale unterbunden. Sie wird rechtlich durch Ausweispapiere (Visa und Pässe), Aufnahmezentren und Abschiebegefängnisse sowie eine ganze Matrix umgrenzter Zeitzonen eingeschränkt (vgl. 
Leutloff-Grandits in diesem Band). Vor allem in wirtschaftlicher Hinsicht haben Grenzen größere Bedeutung erlangt - sie dehnen sich aus und ziehen sich zusammen und folgen dabei den rasanten Veränderungen der Markt- und Sicherheits- sowie der polizeilichen und informationellen Grenzen, die an beliebigen Stellen in der sozialen Matrix auftreten können. Obwohl es heute eine Vielzahl von Grenzen gibt, hat es bislang noch keinen systematischen Versuch gegeben, eine Theorie der Grenze zu entwerfen, die sich für derart unterschiedliche Gebiete als nützlich erweisen könnte. Die Critical Limology will diese Lücke schließen.

Es gibt immer noch eine Menge zu tun, um diesen Ansatz zu entwickeln, aber in der Hoffnung, dass es vorwärtsgeht, habe ich in diesem Beitrag die folgenden vier Argumente aufgestellt.

1. Grenzen unterliegen weder einer universellen Definition noch sollten wir sie auf die modernen empirischen Forschungen reduzieren, sie sind transversale und transhistorische Mobilitätsmuster. Critical Liomology dient der Erforschung des Entstehens, der Mischung und Überlappung dieser Regime in der Geschichte bis in die Gegenwart.

2. Grenzen sind keine Objekte, sie sind Regime wiederkehrender Bewegungen. Critical Limo$\log y$ ist die materiale und geschichtliche Erforschung des gesamten zirkulären Prozesses, der die Grenze als Muster wiederkehrender Bewegungen produziert und reproduziert.

3. Grenzen lassen sich nicht auf Staaten oder staatlich festgeschriebene geografische Gegebenheiten reduzieren. Sie sind vielmehr historisch transversale Muster, die Staatsgrenzen erst produzieren. Critical Limology ist die Erforschung von Grenzen als konstituierende und dinghafte Prozesse, die ihrerseits Gesellschaften produzieren.

4. Grenzen sind nicht durch etwas definiert, was sie sind, sondern durch das, was sie tun und wie sie sich bewegen. Kritische Grenzforschung ist die Erforschung der Art und Weise, wie Grenzregime die Expansion sozialer Mobilitätsmuster vorantreiben, indem sie andere enteignen. Grenzen erlauben soziale Enteignung, um damit sozialer Expansion den Weg zu bereiten.

Aus dem Englischen von Ines Bergfort

\section{Weiterführende Literatur}

Nail, Thomas (2015): The Figure of the Migrant. Stanford: Stanford University Press.

Nail, Thomas 2016): Theory of the Border. Oxford University Press.

Mezzadra, Sandro/Neilson, Brett (2013): Border As Method, Or, the Multiplication of Labor. Durham: Duke University Press.

\section{Literaturverzeichnis}

Alaimo, S./Heckman, S. (Hrsg.) (2008): Material Feminisms. Bloomington, IN: Indiana University Press. Axelsson, L. (2013): Temporalizing the Border. In: Dialogues in Human Geography 3, H. 3, S. 324-326.

Balibar, Etienne (1998): The borders of Europe. In: P. Robbins, Cheah/Robbins, B. (Hrsg.): Cosmopolitics: Thinking and Feeling Beyond the Nation. 1. Aufl., Minneapolis: University of Minnesota Press, S. 216229.

Brown, Wendy (2010): Walled States, Waning Sovereignty. New York: Zone Books.

DHS, Department of Homeland Security, Office of the Inspector General (2009): Immigration and Customs Enforcement's Tracking and Transfers of Detainees. www.oig.dhs.gov/assets/Mgmt/OIG_09-41_M ar09.pdf, 10.4.2015.

Diener, Alexander/Hagen, Joshua (2012): Borders: A Very Short Introduction. New York: Oxford University Press.

Johnson, Corey/Jones, Reece/Paasi, Anssi/Amoore, Louise/Mountz, Alison/Salter, Mark/ Rumford, Chris (2011): Interventions on Rethinking ,the Border ' in Border Studies. In: Political Geography 30, H. 2, S. 619-669. 
Kant, Immanuel (1787/1974): Kritik der reinen Vernunft. 2. Aufl., Frakfurt/M: Suhrkamp. Marx, Karl (1867/1986): Das Kapital, Bd.1. Berlin: Dietz-Verlag.

Nail, Thomas (2015): The Figure of the Migrant. Stanford: Stanford University Press.

Nail, Thomas (2016): Theory of the Border. Oxford, New York: Oxford University Press.

Newman, David (2003): Boundaries. In: Agnew, John/Mitchell, Katharyne/Toal, Gerard (Hrsg.): A Companion to Political Geography. Malden, MA: Blackwell Publishers, S. 123-131.

Nyers, Peter (2012): Moving Borders: The Politics of Dirt. In: Radical Philosophy 174, S. $2-6$.

Simpson, Audra (2014): Mohawk Interruptus: Political Life Across the Borders of Settler States. Durham: Duke University Press.

Williams, William Carlos (1946/1995): Paterson. New York: New Directions Publishing. 